\title{
Exchange bias associated with magnetic glass state in $\mathbf{G d}_{5} \mathbf{G e}_{\mathbf{4}}$
}

\author{
Shujuan Yuan, ${ }^{1, a)}$ Junyi Ge, ${ }^{1}$ Shixun Cao, ${ }^{1}$ Liming $\mathrm{Yu},{ }^{1}$ Anhua $\mathrm{Wu},{ }^{2}$ and Jun $\mathrm{Xu}^{2}$ \\ ${ }^{1}$ Department of Physics, Shanghai University, Shanghai 200444, China \\ ${ }^{2}$ Shanghai Institute of Ceramics, Chinese Academy of Sciences, Shanghai 200050, China
}

(Presented 22 January 2010; received 27 October 2009; accepted 21 December 2009; published online 21 April 2010)

\begin{abstract}
The exchange bias phenomena associated with the magnetic glass state at low temperatures in $\mathrm{Gd}_{5} \mathrm{Ge}_{4}$ are studied. The exchange bias effect originates from the exchange coupling between the ferromagnetic islands and antiferromagnetic matrix in the magnetic glass state. It is found that the exchange bias in $\mathrm{Gd}_{5} \mathrm{Ge}_{4}$ is strongly dependent on the measuring field and the cooling field, which indicates that the antiferromagnetic matrix can be easily transformed into ferromagnetic phase under the applied field. The temperature dependence of the exchange bias field shows that the fluctuation of the temperature has less effect on the exchange bias in the low temperature range. (c) 2010 American Institute of Physics. [doi:10.1063/1.3364050]
\end{abstract}

\section{INTRODUCTION}

As a parent compound of the family of giant magnetocaloric $\mathrm{Gd}_{5}\left(\mathrm{Si}_{\mathrm{x}} \mathrm{Ge}_{1-\mathrm{x}}\right)_{4}$ alloys, $\mathrm{Gd}_{5} \mathrm{Ge}_{4}$ has generated much interest in the physical properties of this compound. ${ }^{1-3}$ $\mathrm{Gd}_{5} \mathrm{Ge}_{4}$ is antiferromagnetic (AFM) below $T_{\mathrm{N}} \sim 128 \mathrm{~K}$. This AFM ground state is metastable and can be transformed into ferromagnetic (FM) state depending on temperature, magnetic field and pressure. ${ }^{4-6}$ A novel glasslike kinetically arrested state was revealed in $\mathrm{Gd}_{5} \mathrm{Ge}_{4}$ at low temperatures. ${ }^{6,7}$ This magnetic glass state is distinctly different from conventional spin glasses where the spin configuration consists of individual spins frozen randomly in the microscopic scale. ${ }^{8}$ The low-temperature, low-field magnetic glass state in $\mathrm{Gd}_{5} \mathrm{Ge}_{4}$ attains a configuration consisting of a small fraction of transformed (equilibrium) FM phase in an untransformed (nonequilibrium) AFM matrix. ${ }^{6}$ Thus, the intrinsic phase separation leads to the FM/AFM interfaces in $\mathrm{Gd}_{5} \mathrm{Ge}_{4}$. Therefore, it would be interesting to explore if exchange bias could exist in the intrinsically phase-separated $\mathrm{Gd}_{5} \mathrm{Ge}_{4}$ compound. Moreover, the exchange bias properties can help to further reveal the magnetic glass state in $\mathrm{Gd}_{5} \mathrm{Ge}_{4}$.

In this work, exchange bias properties associated with magnetic glass state at low temperatures in $\mathrm{Gd}_{5} \mathrm{Ge}_{4}$ are studied. It is found that exchange bias in $\mathrm{Gd}_{5} \mathrm{Ge}_{4}$ is strongly dependent on the measuring field and the cooling field due to the influence of the magnetic field on the relative proportion of the coexisting phases. The results confirm that the metastable AFM matrix can be easily converted into FM phase under the applied field.

\section{EXPERIMENTS}

The $\mathrm{Gd}_{5} \mathrm{Ge}_{4}$ alloy was prepared by arc-melting a mixture of constituent elements Gd (99.9 at. \% purity) and Ge (99.99 at. \% purity) in an argon atmosphere under ambient pressure. The alloy was arc melted five times, with the button being turned over each time to ensure alloy homogeneity. The as-

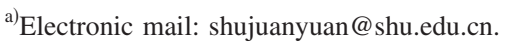

cast $\mathrm{Gd}_{5} \mathrm{Ge}_{4}$ was characterized by $\mathrm{x}$-ray powder diffraction and was found to be a single-phase material. Magnetization measurements were performed using a commercial vibrating sample magnetometer (Quantum Design, PPMS-VSM).

\section{RESULTS AND DISCUSSION}

The hysteresis loops of $\mathrm{Gd}_{5} \mathrm{Ge}_{4}$ at $5 \mathrm{~K}$ with both the zero-field cooling (ZFC) and field-cooling (FC) processes were measured. For the ZFC process, the sample was cooled in zero magnetic field from room temperature to $5 \mathrm{~K}$. For the FC process, the sample was cooled in 3000 Oe magnetic field from room temperature to $5 \mathrm{~K}$. Then, the hysteresis loops were measured between \pm 3000 Oe. As shown in Fig. 1 , the ZFC loop is symmetric around zero point, whereas the FC loop shifts both to the negative field and to the positive magnetization. The magnitude of the exchange bias effect observed in the samples is usually compared quantitatively using the exchange bias field $H_{E}$ and the coercivity field $H_{C}$. It can be seen that the exchange bias field $H_{E}$ of $\mathrm{Gd}_{5} \mathrm{Ge}_{4}$ is about $7 \mathrm{Oe}$, which is much smaller than other intrinsically phase-separated cobaltites and manganites. ${ }^{9,10}$ It might originate from the special spin configuration in the magnetic glass

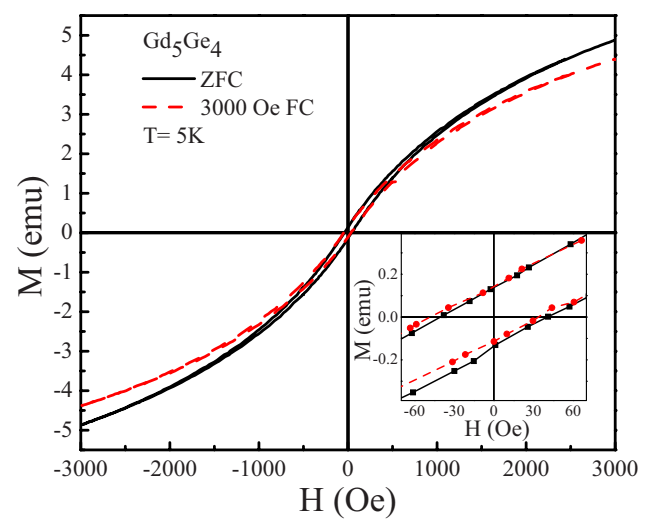

FIG. 1. (Color online) Hysteresis loops of $\mathrm{Gd}_{5} \mathrm{Ge}_{4}$ at $5 \mathrm{~K}$ measured after $\mathrm{ZFC}$ and FC in 3000 Oe field. Inset shows the enlarged view of the low field region. 


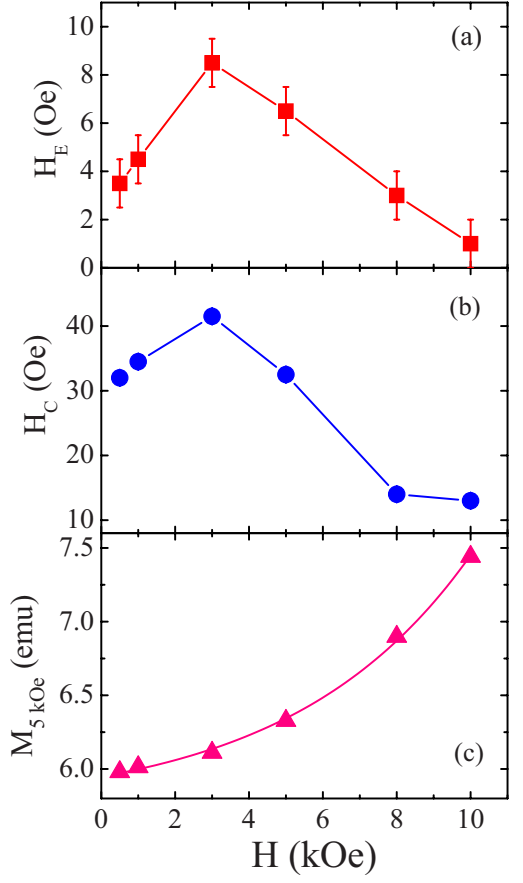

FIG. 2. (Color online) Cooling field dependence of (a) $H_{E}$, (b) $H_{C}$, and (c) $M_{5} \mathrm{kOe}$ for $\mathrm{Gd}_{5} \mathrm{Ge}_{4}$ at $10 \mathrm{~K}$ after field cooled from room temperature to 10 $\mathrm{K}$. The measuring filed is between $\pm 5 \mathrm{kOe}$.

state of $\mathrm{Gd}_{5} \mathrm{Ge}_{4}$. Regions (maybe in micrometer scale or less) with long-range FM and AFM ordering are frozen randomly in the magnetic glass state. ${ }^{6}$ The large size of FM islands will weaken the exchange bias effect, leading to the small value of $H_{E}$.

The AFM ground state of $\mathrm{Gd}_{5} \mathrm{Ge}_{4}$ in low temperature is metastable and can be transformed into the equilibrium FM state depending on the temperature and the applied magnetic field. ${ }^{4-6}$ Therefore, it is necessary to explore the effect of magnetic field and temperature on the exchange bias in $\mathrm{Gd}_{5} \mathrm{Ge}_{4}$. First, we studied the influence of cooling field on the exchange bias. After FC process under different applied fields from room temperature to $10 \mathrm{~K}$, the loops were measured between $\pm 5 \mathrm{kOe}$. Figure 2 shows the cooling field dependence of the loop parameters, including the exchange bias field $H_{E}$, the coercivity $H_{C}$, and magnetization at $H$ $=5 \mathrm{kOe} M_{5} \mathrm{kOe}$.

The exchange bias field $H_{E}$ and the coercivity $H_{C}$ both increase and then decrease with the increase in cooling field. The exchange bias field approaches zero when the cooling field is larger than $10 \mathrm{kOe} . M_{5} \mathrm{kOe}$ increases with the cooling field by the exponential growth, indicating the FM islands increase with the increase in the cooling field. At small cooling fields, $H_{E}$ and $H_{C}$ increase with increasing cooling field, whereas $M_{5} \mathrm{kOe}$ increases a little. The little change in $M_{5}$ kOe indicates that the proportion of FM islands changes little at small cooling fields. With the increase in cooling field, the alignment degree of the proportion of FM islands along a preferential direction is enhanced, which reduces the effect of an averaging of the anisotropy due to randomness. Therefore, $H_{E}$ and $H_{C}$ increase with increasing cooling field. At high cooling field, not only the alignment degree of the moments of FM islands is enhanced but also the size of FM

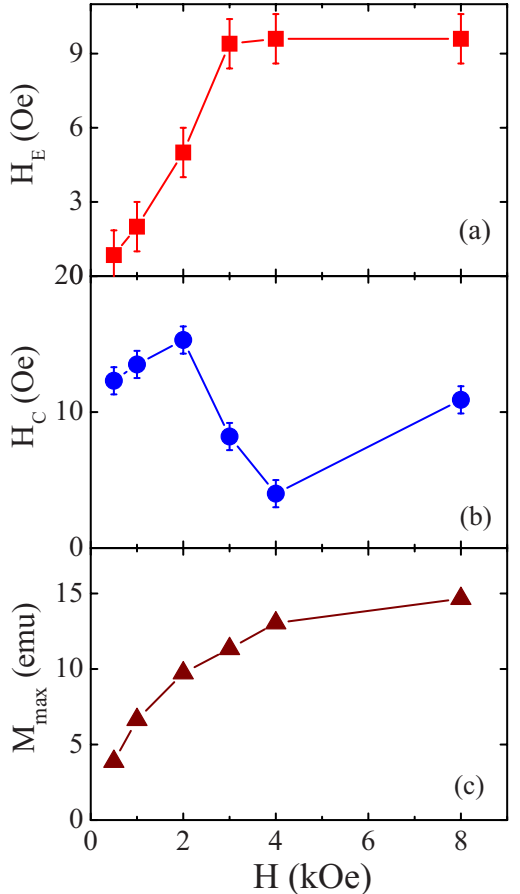

FIG. 3. (Color online) Measuring field dependence of (a) $H_{E}$, (b) $H_{C}$, and (c) $M_{\max }$ for $\mathrm{Gd}_{5} \mathrm{Ge}_{4}$ at $10 \mathrm{~K}$. The cooling field is $8 \mathrm{kOe}$.

islands increases. As the FM islands grow up, exchange bias is reduced. The similar phenomena were observed in other phase-separated perovskite cobaltites and manganites. ${ }^{9,10}$ This effect is qualitatively analogous to that in FM/AFM bilayers where exchange bias field is inversely proportional to the thickness of the FM layer and becomes zero when the FM layer is too thick.

Then, we studied the influence of measuring field on the exchange bias in $\mathrm{Gd}_{5} \mathrm{Ge}_{4}$. For each measurement, the sample was cooled in a field of $8 \mathrm{kOe}$ from room temperature to 10 $\mathrm{K}$, and then the hysteresis loops were measured between $\pm 0.5, \pm 1, \pm 2, \pm 3, \pm 5$, and $\pm 8 \mathrm{kOe}$, respectively. As shown in Fig. 3, the exchange bias field $H_{E}$ increases with the increase in measuring field and tends to saturate when the applied field is larger than $3 \mathrm{kOe} . H_{C}$ first increases and then decreases with increasing the measuring field, but doesn't show obvious change. $M_{\max }$ is the magnetization value obtained at the maximum of the measuring field. $M_{\max }$ increases with increasing the measuring field and tends to saturate at high measuring field, which reflects the magnetized state of the FM region. When the measuring field is low, it is not strong enough to have all the FM islands fully magnetized. $H_{E}$ will increase with the increase in the measuring field. When the FM region can be fully magnetized, $H_{E}$ will tend to saturate.

The temperature dependence of the exchange bias properties is also studied. In these measurements, the sample was cooled down from room temperature to the measuring temperature with an applied field $H_{\text {cool }}=3 \mathrm{kOe}$. Once the measuring temperature was reached, the hysteresis loop was measured between $\pm 3 \mathrm{kOe}$, and then rise to room temperature. This process was repeated for every measuring temperature. The temperature dependence of $H_{E}$ is shown in Fig. 4. $H_{E}$ does not show a drastic change in the temperature 


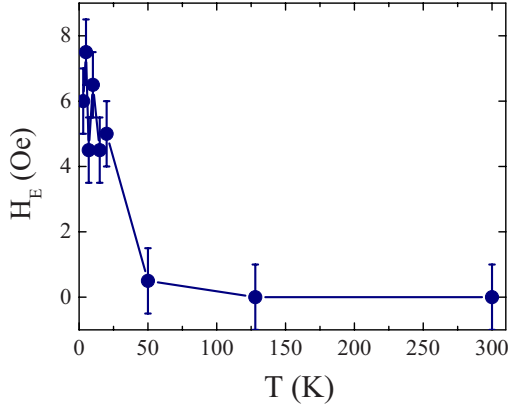

FIG. 4. (Color online) Temperature dependence of $H_{E}$. The cooling field is $3 \mathrm{kOe}$ and the measuring filed is between $\pm 3 \mathrm{kOe}$.

range from 3 to $20 \mathrm{~K}$. As previously reported, the magnetic glass state exists in this temperature range, ${ }^{6}$ which will contribute to the exchange bias effect. According to the effect of temperature cycling on $\mathrm{Gd}_{5} \mathrm{Ge}_{4}$, the frozen metastable AFM ground state can be gradually converted to the FM state due to the energy fluctuations below $\sim 20 \mathrm{~K} .{ }^{6}$ However, this effect cannot be obviously reflected by the $H_{E}$, which indicates that exchange bias effect cannot reflect the AFM-FM transition induced by temperature. As the temperature is above 50 $\mathrm{K}$, the exchange bias vanishes.

\section{CONCLUSIONS}

In conclusion, we discovered exchange bias phenomena associated with magnetic glass state at low temperature in $\mathrm{Gd}_{5} \mathrm{Ge}_{4}$. It is found that exchange bias in $\mathrm{Gd}_{5} \mathrm{Ge}_{4}$ is strongly dependent on the measuring field and the cooling field. The cooling field dependence of the exchange bias indicates that the metastable AFM ground state can be easily transformed into FM phase under the applied field. $H_{E}$ does not show a drastic change in the temperature range from 3 to $20 \mathrm{~K}$, which indicates that the exchange bias effect cannot reflect the reversible or irreversible AFM-FM transition induced by temperature.

\section{ACKNOWLEDGMENTS}

This work was supported by the National Natural Science Foundation of China (Grant No. 50932003), the Science and Technology Innovation Fund of the Shanghai Education Committee (Grant No. 09ZZ95), and the Science and Technology Committee of Shanghai Municipality (Grant Nos. 08dj1400202 and 10ZR1411000).

${ }^{1}$ V. K. Pecharsky and K. A. Gschneidner, Jr., Phys. Rev. Lett. 78, 4494 (1997).

${ }^{2}$ H. Tang, V. K. Pecharsky, K. A. Gschneidner, Jr., and A. O. Pecharsky, Phys. Rev. B 69, 064410 (2004).

${ }^{3}$ Z. W. Ouyang, H. Nojiri, S. Yoshii, G. H. Rao, and Y. C. Wang, Phys. Rev. B 80, 054401 (2009).

${ }^{4}$ L. Morellon, J. Blasco, P. A. Algarabel, and M. R. Ibarra, Phys. Rev. B 62, 1022 (2000).

${ }^{5}$ C. Magen, Z. Arnold, L. Morellon, Y. Skorokhod, P. A. Algarabel, M. R. Ibarra, and J. Kamarad, Phys. Rev. Lett. 91, 207202 (2003).

${ }^{6}$ S. B. Roy, M. K. Chattopadhyay, P. Chaddah, J. D. Moore, G. K. Perkins, L. F. Cohen, K. A. Gschneidner, Jr., and V. K. Pecharsky, Phys. Rev. B 74, 012403 (2006).

${ }^{7}$ J. D. Moore, G. K. Perkins, K. Morrison, L. Ghivelder, M. K. Chattopadhyay, S. B. Roy, P. Chaddah, K. A. Gschneidner, Jr., V. K. Pecharsky, and L. F. Cohen, J. Phys.: Condens. Matter 20, 465212 (2008).

${ }^{8}$ J. A. Mydosh, Spin-Glasses (Taylor \& Francis, London, 1992).

${ }^{9}$ Y. K. Tang, Y. Sun, and Z. H. Cheng, Phys. Rev. B 73, 174419 (2006).

${ }^{10}$ S. Karmakar, S. Taran, E. Bose, B. K. Chaudhuri, C. P. Sun, C. L. Huang, and H. D. Yang, Phys. Rev. B 77, 144409 (2008). 